\title{
Generalized Anticipation Effect Models for Traffic Flow
}

\author{
Liuhua Zhu',2 \\ ${ }^{1}$ School of Physics and Telecommunication Engineering, Yulin Normal University, Yulin, China \\ ${ }^{2}$ Guangxi Colleges and Universities Key Laboratory of Complex System Optimization and Big Data Processing, Yulin, China \\ Email: zhuliuhua421@sina.com
}

How to cite this paper: Zhu, L.H. (2020) Generalized Anticipation Effect Models for Traffic Flow. Journal of Applied Mathematics and Physics, 8, 367-374. https://doi.org/10.4236/jamp.2020.83028

Received: January 27, 2020

Accepted: February 24, 2020

Published: February 27, 2020

Copyright (c) 2020 by author(s) and Scientific Research Publishing Inc. This work is licensed under the Creative Commons Attribution International License (CC BY 4.0).

http://creativecommons.org/licenses/by/4.0/

\begin{abstract}
Taking into account the startup behaviour of following vehicles in the waiting area of urban traffic signal, we propose an extended Nagel-Schreckenberg model for single-lane traffic flow, in which the dynamic behavior of each vehicle depends on not only its own headway but also the headway of the immediately preceding one. The numerical simulation of the present model reproduces some complicated nonlinear phenomena observed in real traffic such as free flow, ghostly blockage, synchronized flow and so on. For specific parameter combinations the flow-density relation of this model shows two meta-stable branches near the transition density from free flow to wide moving traffic jam. Finally, the analytical results of the model under some specific parameters are given by using the mean field theory.
\end{abstract}

\section{Keywords}

Traffic Flow, Cellular Automaton, Discrete Model, Anticipation Effect, Meta-Stable State

\section{Introduction}

The problem of traffic congestion is becoming more and more serious, which seriously affects human life and restricts the development of society. Traffic congestions are occurring daily in the metropolis of the world, wasting energy and time. In order to alleviate traffic congestions, engineers and technicians have experienced a long exploration process. In the last few decades, the traffic problem has attracted extensive attention of scientists from different fields, because of various nonlinear dynamic phenomena and non-equilibrium phase transitions observed in the real traffic. Different types of models have been proposed to simulate real traffic systems, such as the hydrodynamic model based on con- 
tinuity description, the gas-kinetic model based on statistics description, and the cellular automaton model based on discreteness description. Among these models, the cellular automaton model has become one of the most widely used models because of its remarkable simplicity of rules and high efficiency of computation [1]-[6].

The first cellular automata model for single-lane traffic flow was proposed by Nagel and Schreckenberg [1], which successfully reproduces some nonlinear phenomena in the real traffic, such as the start-and-stop wave and the ghostly congestion. Later, the original model was widely extended and different variant models were proposed one after another [7] [8] [9]. The variant models are capable of simulating traffic flow more realistically.

The rest of this paper is organized as follows. In Section 2, some basic notions on the generalized anticipation effect model are introduced. Then in Section 3, we give the main results of numerical simulations. The dynamics of the anticipation effect model with specific parameters are numerically simulated and theoretically analyzed in Section 4 . Section 5 is devoted to the concluding remarks, in which we point out the application prospect of this model in traffic management.

\section{Basic Notions on the Generalized Anticipation Effect Model}

Drivers always try their best to observe the headway of the nearest vehicle in front of them, rather than being limited to their own headway, especially driving on a straight road or crossing an uncontrolled zebra-stripe. We all have the experience that when the intersection signal light turns green, the following vehicles will even start before the vehicles in front. The reason lies in the fact that the vehicle ahead will start within the headway and will not cause a collision. The impact of the anticipation effect on the transportation system naturally arouses our interest. In this paper, we propose a generalized traffic flow model with anticipation effect [10] [11], in which the appropriate adjustment of relevant parameters can simulate different traffic scenes.

In the model, the road is divided into cells of length $7.5 \mathrm{~m}$, which is interpreted as the length of a vehicle plus the gap between vehicles in a jam. Each cell can either be empty or occupied by only one vehicle. The state of the ith vehicle is characterised by its instantaneous velocity $v_{i}$ and position $x_{i}$. The present model is a probabilistic cellular automaton, in which the velocities and positions of vehicles can only take a series of discrete values. The dynamics of the present model are defined by the following set of rules, which are applied to all vehicles at each time-step,

1) Acceleration or deceleration:

$$
v_{i}(t+1 / 2)=\min \left\{v_{\max }, v_{i}(t)+1,\left\lceil\alpha d_{i}(t)+\beta v_{i+1}^{*}(t+1)\right]\right\}
$$

2) Negative compensation of velocity: 


$$
\begin{aligned}
& p=\left\lceil\alpha d_{i}(t)+\beta v_{i+1}^{*}(t+1)\right\rceil-\left(\alpha d_{i}(t)+\beta v_{i+1}^{*}(t+1)\right) \\
& \text { for } \alpha d_{i}(t)+\beta v_{i+1}^{*}(t+1)<v_{\max } \\
& \text { or } p=0 \text { for } \alpha d_{i}(t)+\beta v_{i+1}^{*}(t+1) \geq v_{\max } \\
& v_{i}(t+1)=\max \left(0, v_{i}(t+1 / 2)-1\right) \text { with probability } p
\end{aligned}
$$

3) Updating of position:

$$
x_{i}(t+1)=x_{i}(t)+v_{i}(t+1)
$$

Here the parameters $\alpha$ and $\beta$ are primary and secondary expectation factors, respectively. The expression $\lceil x\rceil$ represents the smallest integer not less than $x$, and $v_{i+1}^{*}(t+1)$ is a virtual velocity of the $(i+1)$ th vehicle at the time step $(t+1)$, given as

$$
v_{i+1}^{*}(t+1)=\min \left\{v_{\max }-1, v_{i+1}(t), \max \left(0, d_{i+1}(t)-1\right)\right\}
$$

\section{Main Results}

In the numerical simulation of traffic flow, the maximum velocity is set to $v_{\max }=5$, which corresponds to $135 \mathrm{~km} / \mathrm{h}$, just as the normal free-flow velocity on a motorway. For simplicity, in this paper only one type of vehicle is considered and therefore the same maximum velocity will be used for all vehicles. The system size is set to $L=1000$, because the results described below do not change qualitatively for larger size. The periodic boundary condition is adopted, so the density, $\rho=N / L$, remains unchanged with time, where $N$ is the number of vehicles.

In this paper, two types of initialization methods are adopted. One is the random initialization, where $N$ vehicles are randomly distributed in the cells, and the velocity of each vehicle is randomly selected from the set $\left\{0,1,2, \cdots, v_{\max }\right\}$. The other is the homogeneous initialization, in which the vehicles are arranged equally, and the velocity of each vehicle is the same.

The fundamental diagram describes the dependence of vehicle flow on density, and is often used to evaluate the rationality of the model. From Figure 1 the impact of the primary and secondary expectation factors on traffic flow can be observed. The simulation results show that when one of the two parameters is fixed, the maximum flow will increase with the increase of the other parameter. By comparing Figure 1(a) with Figure 1(b), one may notice that the influence of the primary expectation factor on traffic flow is stronger than that of the secondary factor.

From Figure 2, one may find that the system displays free flow at a low density while start-and-stop waves at a high density, which is consistent with the real traffic situation. In Figure 2(a), all vehicles drive at the desired velocity, and the whole system presents a smooth moving phase. However, in Figure 2(b), the headway in front of the vehicle is not enough to meet the expected velocity, thus the movement of the vehicle is limited, resulting in ghostly congestions.

Previous studies have confirmed that the initial state plays a vital role in system evolution. For each panel in Figure 3, we have computed two flow diagrams, 


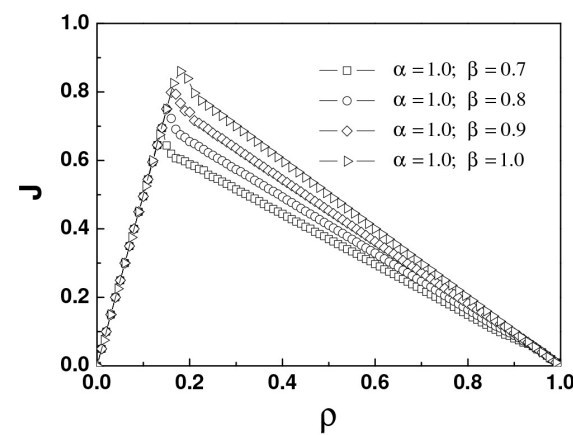

(a)

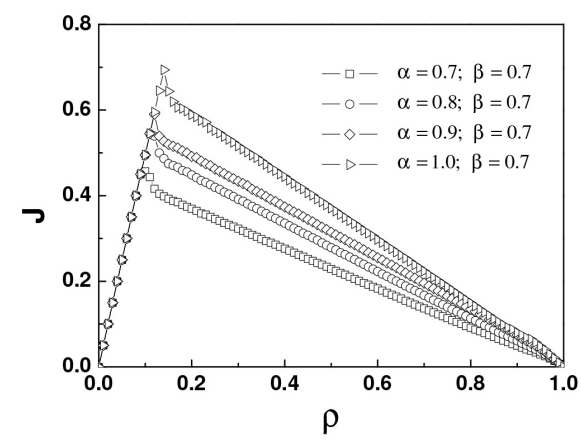

(b)

Figure 1. The fundamental diagrams for different primary and secondary expectation factors. If one of the two factors is fixed, the maximum flow will increase with the increase of the remaining parameter.

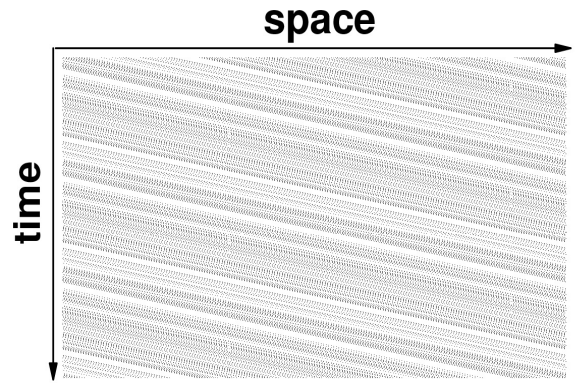

(a)

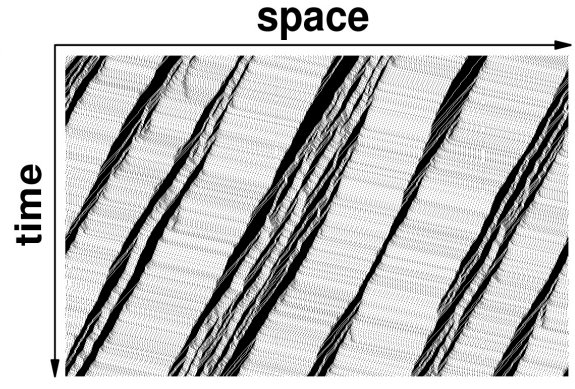

(b)

Figure 2. The spatial-temporal diagrams of the present model for different traffic densities. (a) $\rho=0.1$, (b) $\rho=0.3$. The vehicles are moving from left to right. A vehicle is represented by a black dot. The time axis is vertical down. Other parameters are set to $\alpha=0.9$ and $\beta=0.7$. Both of them come from a random initial state, in which the transient process has been discarded.

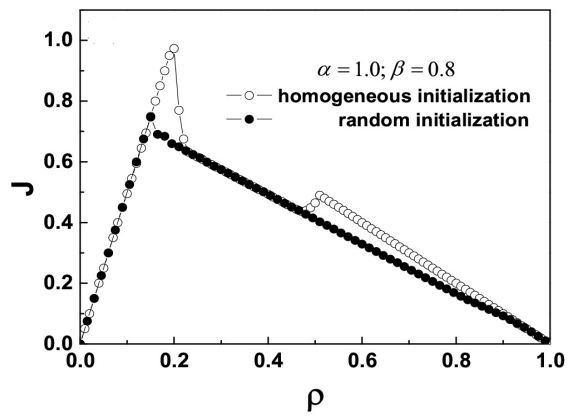

(a)

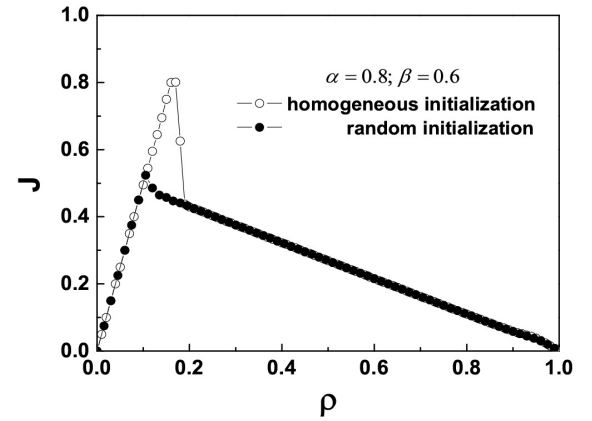

(b)

Figure 3. The hysteresis loops in the fundamental diagrams. The upper branch is obtained from a homogeneous initial state while the lower branch from a random initial configuration. The parameters are set to $\alpha=1.0$ and $\beta=0.8$ in the panel (a) and $\alpha=0.8$ and $\beta=0.6$ in the panel (b).

$J(\rho)$, labeled as homogeneous and random initializations. The upper branch of the fundamental diagram corresponds to the calculation starting with a homo- 
geneous initialization, while the lower branch is obtained starting from a initially random configuration. Because the traffic flow depends on the origin of the system, the two fundamental diagrams are also called hysteresis loops.

The sensitivity of spatial-temporal evolution to initial states is displayed in Figure 4. At the same density, started from the two different initial configurations, Figure 4(a) and Figure 4(b) show two different steady states. In Figure 4(a), the vehicles move in step from left to right at the same speed. However, in Figure $4(\mathrm{~b})$, the initial random distribution of vehicles breaks the uniform and orderly pace of the system, traffic jams appear in advance.

Compared with the velocity effect model proposed in Ref.10, the model presented in this paper is more flexible, which can adjust the expectation factors in real time according to the actual traffic conditions. Different from the static adaptive cruise control model proposed in [11], our model is dynamic, which considers the anticipatory behavior of the preceding vehicle and reproduces the meta-stable phenomenon in the real traffic system.

\section{Theoretical Analysis}

In this section, we concentrate on the special case of $\alpha=\beta=1.0$. In view of $\alpha=\beta=1.0$, the expected velocity of each vehicle is always an integer, resulting in no negative compensation for the velocity. For the random initialization, the running state of the vehicles is equivalent to the deterministic $\mathrm{Na}$ gel-Schreckenberg model. Therefore, the relationship between flow and density can be written as [1],

$$
J=\min \left(\rho v_{\max }, 1-\rho\right)
$$

While for the initial homogeneous configuration, every vehicle is in step. This state of synchronization will not be broken until the ith vehicle cannot keep moving at $v_{\max }$. Therefore, when the system reaches the critical point, the following conditions should be satisfied.

\section{space}

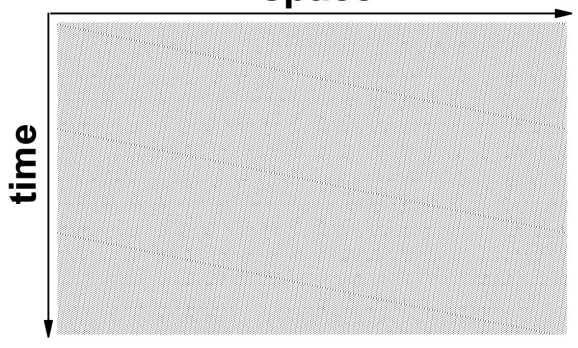

(a)

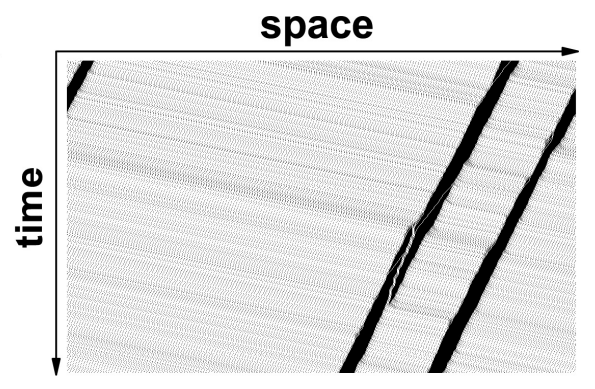

(b)

Figure 4. The sensitivity of spatial-temporal evolution to initial states at the same traffic density $\rho=0.15$. (a) Homogeneous initialization; (b) Random initialization. Other parameters are set to $\alpha=0.8$ and $\beta=0.6$. The vehicles are moving from left to right. A vehicle is represented by a black dot. The time axis is vertical down. The transient process has been discarded. 


$$
d+(d-1)=v_{\max }
$$

where $d$ is the space interval between adjacent vehicles. Thus, the critical density of the system is obtained,

$$
\rho_{c}=\frac{1}{d+1}=\frac{2}{v_{\max }+3}=\frac{1}{4}
$$

If the vehicle is expected to travel at an integer velocity, the value of $d$ can only be $1,2,3$. The point of $d=3$ corresponds to the critical point of the system. The point of $d=2$ constitutes a turning point of the system, after which the traffic flow drops sharply. When $d=1$, the anticipation effect no longer works, the relationship between flow and density is the same as that of the deterministic asymmetric exclusive process. To summarize, the relationship between flow and density for homogeneous initialization is given by,

$$
J= \begin{cases}5 \rho & 0<\rho \leq \frac{1}{4} \\ -3.25 \rho+2.0625 & \frac{1}{4}<\rho \leq \frac{1}{3} \\ -\rho+1 & \frac{1}{3}<\rho \leq 1 .\end{cases}
$$

The results show that the flow-density diagram produced by the numerical simulation is consistent with that obtained from the theoretical analysis, as shown in Figure 5(a). Figure 5(b) shows that for the homogeneous initialization, the uniform maximum velocity of the vehicles can be maintained to a higher density. However, when the average velocity of the system drops below 3 , the impact of the initial configurations is no longer effective, resulting in the overlapping of the two curves.

The four panels in Figure 6 clearly show the detailed characteristics of velocity evolution of vehicles under different traffic densities. Keeping pace as much as possible is the eternal feature of the homogeneous initialization.

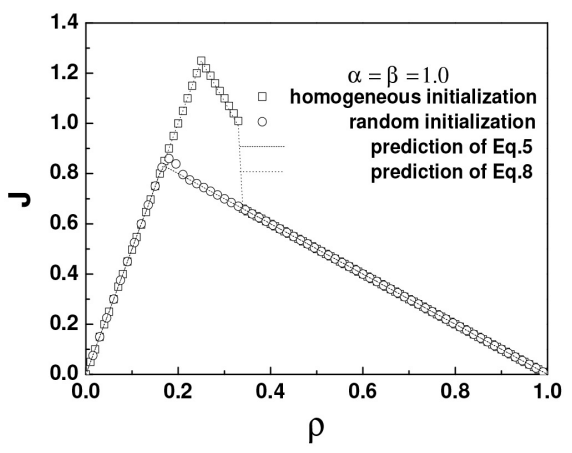

(a)

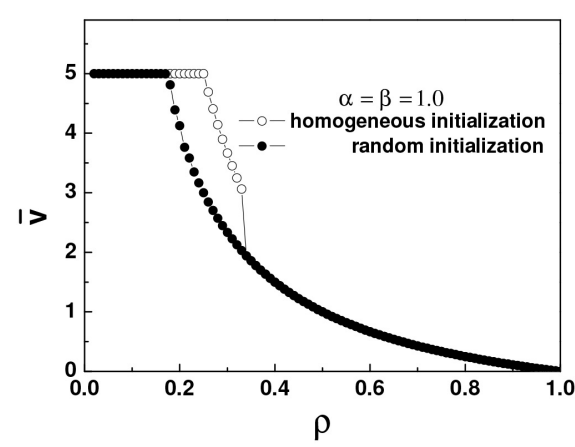

(b)

Figure 5. Traffic characteristics of the model in the case of $\alpha=\beta=1.0$. (a) The fundamental diagrams for different initial configurations; (b) The relationship between average velocity and traffic density for different initial configurations. 


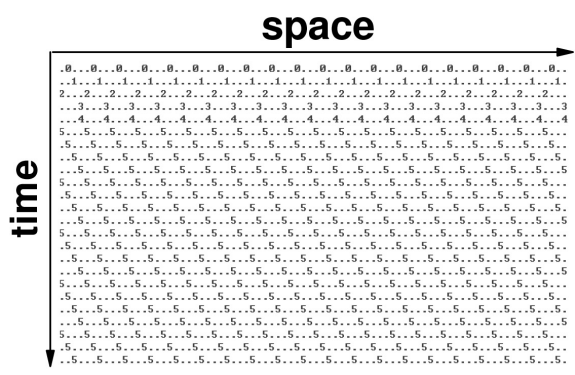

(a)

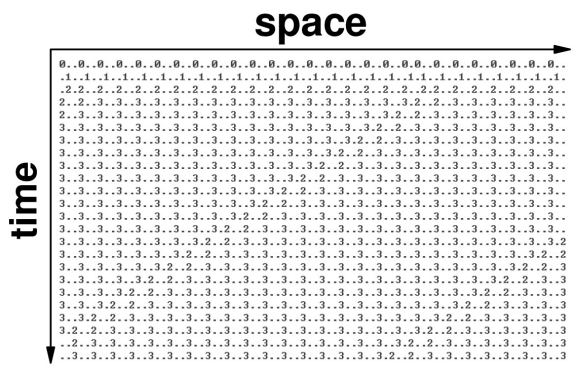

(c)

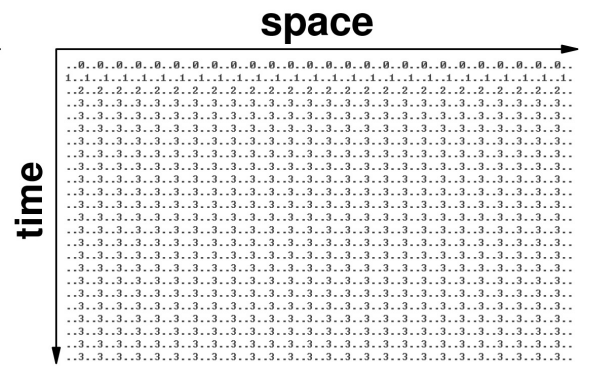

(b)

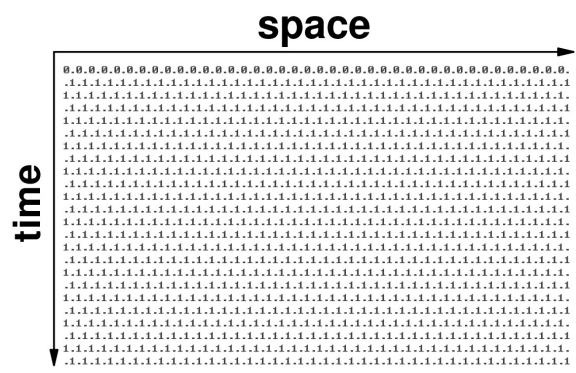

(d)

Figure 6. Snapshots of the velocity evolution starting with a homogeneous initialization for different traffic densities. (a) $\rho=0.25$, (b) $\rho=0.33$, (c) $\rho=0.34$, (d) $\rho=0.50$. The time steps are set to $1: 24$ and space positions are set to $500 \sim 579$.

\section{Conclusion}

The considerations in this paper show the flexibility of the cellular automaton approach to traffic flow problems. A rather simple and natural extension of the rules of the Nagel-Schreckenberg model allows us to describe the formation of meta-stable states in the fundamental diagram. The results presented here have potential applications. In the traffic management and control practice, one of the most practical tasks is to stabilize the homogeneous branch of the fundamental diagram so as to maximize traffic flow without increasing the infrastructure. This can be done using signals to control the inflow, as in the case of the Lincoln tunnel in New York [12]. In the traffic control of intersections, a considerable increase of the maximum capacity can be achieved for an optimal combination of the red-green-signal periods. The optimal intervals of the signal induce the metastable behavior of the traffic system. How to set the green-time-ratio scientifically and effectively is the focus of our next work.

\section{Acknowledgements}

This work is supported by the National Natural Science Foundation of China under Grant Nos. 61563054 and 11875031, the Natural Science Foundation of Guangxi under Grant No. 2019JJA110069, the open fund of Guangxi Colleges and Universities Key Laboratory of Complex System Optimization and Big Data Processing under Grant No. 2015CSOBDP0101, Initiation Fund of Doctoral Research of Yulin Normal University under Grant No. G20150003. 


\section{Conflicts of Interest}

The author declares no conflicts of interest regarding the publication of this paper.

\section{References}

[1] Nagel, K. and Schreckenberg, M. (1992) A Cellular Automaton Model for Freeway Traffic. Journal of Physics I France, 2, 2221-2229. https://doi.org/10.1051/jp1:1992277

[2] Barlovic, R., Santen, L., Schadschneider, A., et al. (1998) Metastable States in Cellular Automata for Traffic Flow. The European Physical Journal B-Condensed Matter and Complex Systems, 5, 793-800. https://doi.org/10.1007/s100510050504

[3] Nishinari, K., Fukui, M. and Schadschneider, A. (2004) A Stochastic Cellular Automaton Model for Traffic Flow with Multiple Metastable States. Journal of Physics A: Mathematical and General, 37, 3101. https://doi.org/10.1088/0305-4470/37/9/003

[4] Larraga, M.E. and Alvarez-Icaza, L. (2014) Cellular Automata Model for Traffic Flow with Safe Driving Conditions. Chinese Physics B, 23, Article ID: 050701. https://doi.org/10.1088/1674-1056/23/5/050701

[5] Zamith, M., Leal-Toledo, R.C.P., Clua, E., et al. (2015) A New Stochastic Cellular Automata Model for Traffic Flow Simulation with Drivers' Behavior Prediction. Journal of Computational Science, 9, 51-56. https://doi.org/10.1016/j.jocs.2015.04.005

[6] Hou, G.Y. and Chen, S.R. (2019) An Improved Cellular Automaton Model for Work Zone Traffic Simulation Considering Realistic Driving Behavior. Journal of the Physical Society of Japan, 88, Article ID: 084001. https://doi.org/10.7566/JPSJ.88.084001

[7] Xiao, X.P., Yang, J.W., Mao, S.H., et al. (2017) An Improved Seasonal Rolling Grey Forecasting Model Using a Cycle Truncation Accumulated Generating Operation for Traffic Flow. Applied Mathematical Modelling, 51, 386-404.

https://doi.org/10.1016/j.apm.2017.07.010

[8] Malecki, K. (2018) A Computer Simulation of Traffic Flow with On-Street Parking And Drivers?? Behaviour Based on Cellular Automata and a Multi-Agent System. Journal of Computational Science, 28, 32-42. https://doi.org/10.1016/j.apm.2017.07.010

[9] Kuang, H., Wang, M.T., Lu, F.H., et al. (2019) An Extended Car-Following Model Considering Multi-Anticipative Average Velocity Effect under V2V Environment. Physica A: Statistical Mechanics and Its Applications, 527, Article ID: 121268. https://doi.org/10.1016/j.physa.2019.121268

[10] Li, X.B. Wu, Q.S. and Jiang, R. (2001) Cellular Automaton Model Considering the Velocity Effect of a Car on the Successive Car. Physical Review E, 64, Article ID: 066128. https://doi.org/10.1103/PhysRevE.64.066128

[11] Jiang, R. and Wu, Q.S. (2006) The Adaptive Cruise Control Vehicles in the Cellular Automata Model. Physics Letters A, 359, 99-102.

https://doi.org/10.1103/PhysRevE.64.066128

[12] Chowdhury, D., Santen, L. and Schadschneider, A. (2000) Statistical Physics of Vehicular Traffic and Some Related Systems. Physics Reports, 329, 199-332. https://doi.org/10.1016/S0370-1573(99)00117-9 